\title{
Correction to: Symmetric quiver Hecke algebras and R-matrices of quantum affine algebras
}

\author{
Seok-Jin Kang ${ }^{1} \cdot$ Masaki Kashiwara $^{2,3}$. \\ Myungho Kim ${ }^{4}$
}

Published online: 31 January 2019

(C) Springer-Verlag GmbH Germany, part of Springer Nature 2019

\section{Correction to: Invent. math. (2018) 211:591-685 https://doi.org/10.1007/s00222-017-0754-0}

Abstract We fix a mistake in [1] on the definition of commuting family of central objects.

Mathematics Subject Classification 81R50, 16G, 16T25, 17B37

We will freely make use of the notations from [1]. Let $\mathcal{T}$ be a tensor category, and let $\left\{P_{i}\right\}_{i \in I}$ be a family of objects in $\mathcal{T}$. In the beginning of Appendix A.6,

The original article can be found online at https://doi.org/10.1007/s00222-017-0754-0.

\footnotetext{
$凶$ Myungho Kim

mkim@khu.ac.kr

Seok-Jin Kang

sjkang@uaeu.ac.ae

Masaki Kashiwara

masaki@ kurims.kyoto-u.ac.jp

1 Department of Mathematical Sciences, United Arab Emirates University, P. O. Box 15551, Al Ain, Abu Dhabi, United Arab Emirates

2 Research Institute for Mathematical Sciences, Kyoto University, Kyoto 606-8502, Japan

3 Korea Institute for Advanced Study, Seoul 02455, Korea

4 Department of Mathematics, Kyung Hee University, Seoul 02447, Korea
} 
we say that $\left\{\left(P_{i}, R_{P_{i}}\right)_{i \in I}\right\}$ is a commuting family of central objects if it satisfies two conditions

(a) for any $i \in I, R_{P_{i}}$ is an isomorphism

$$
R_{P_{i}}(X): P_{i} \otimes X \stackrel{\sim}{\longrightarrow} X \otimes P_{i}
$$

functorial in $X \in \mathcal{T}$ such that

$$
P_{i} \otimes X \otimes Y \underset{R_{P_{i}}(X)}{\stackrel{R_{P_{i}}(X \otimes Y)}{\longrightarrow} X \otimes P_{i} \otimes Y \underset{R_{P_{i}}(Y)}{\longrightarrow}} X \otimes Y \otimes P_{i} \quad \text { commutes for any } X, Y \in \mathcal{T},
$$

(b) $R_{P_{j}}\left(P_{i}\right) \circ R_{P_{i}}\left(P_{j}\right)=\operatorname{id}_{P_{i} \otimes P_{j}}$ for any $i, j \in I$ such that $i \neq j$.

If it further satisfies the condition

$$
R_{P_{i}}\left(P_{i}\right)=\operatorname{id}_{P_{i} \otimes P_{i}} \text { for any } i \in I,
$$

then it was called a strictly commuting family of central objects.

In the next pages, we asserted that if $\left\{\left(P_{i}, R_{P_{i}}\right)_{i \in I}\right\}$ is a commuting family of central objects, then the localization $\widetilde{\mathcal{T}}=\mathcal{T}\left[P_{i}^{\otimes-1} \mid i \in I\right]$ of $\mathcal{T}$ becomes a tensor category with a unit object $(\mathbf{1}, \overrightarrow{0})$. This is incorrect since the following gives a counterexample: Let $\mathcal{T}$ be the category of vector spaces over a field $\mathbf{k}$ and let $P=\mathbf{k}^{\oplus 2}$. Let $R_{P}(X): P \otimes X \rightarrow X \otimes P$ be the map given by $v \otimes x \mapsto x \otimes v$ for $v \in P$ and $x \in X$. Then $\left\{\left(P, R_{P}\right)\right\}$ satisfy the conditions (a) and (b). Since the endomorphism ring

$$
\begin{aligned}
& \operatorname{End}_{\widetilde{\mathcal{T}}}((\mathbf{1}, 0))=\underset{\gamma \in \mathbb{\mathbb { Z }}_{\geq 0}}{\lim _{\mathcal{T}}} \operatorname{End}_{\mathcal{T}}\left(\mathbf{1} \otimes P^{\gamma}\right)
\end{aligned}
$$

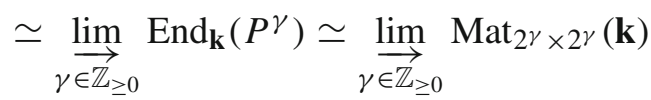

is not commutative, the object $(\mathbf{1}, 0)$ cannot be a unit object of a tensor category ([2, Exercise 4.11 (ii)]).

The problem occurs since we do not assume that the family satisfy condition (A.4). As the simplest remedy, we include condition (A.4) in the definition of commuting family of central objects from the beginning and remove the definition of strictly commuting family of central objects in the paper. Under the new definition of commuting family of central objects, we can construct the category $\mathcal{T}\left[P_{i}^{\otimes-1} \mid i \in I\right]$ as well as the category $\mathcal{T}\left[P_{i} \simeq \mathbf{1} \mid i \in I\right]$ in the graded case (Appendix A.7). All the arguments in the main body of our paper also work without any problem. 


\section{References}

1. Kang, S.-J., Kashiwara, M., Kim, M.: Symmetric quiver Hecke algebras and $R$-matrices of quantum affine algebras. Invent. Math. 211, 591-685 (2018)

2. Kashiwara, M., Schapira, P.: Categories and Sheaves, Grundlehren der mathematischen Wissenschaften 332. Springer, Berlin (2006)

Publisher's Note Springer Nature remains neutral with regard to jurisdictional claims in published maps and institutional affiliations. 